\title{
Estudos clínicos randomizados dos benefícios e limitações do Irradiation Laser Intravenous of Blood (ILIB) na Odontologia: revisão integrativa
}

\author{
Randomized clinical studies of the benefits and limitations of Irradiation Laser Intravenous of \\ Blood (ILIB) in Dentistry: an integrative review \\ Clínicos aleatorizados de los beneficios y limitaciones de la Irradiation Laser Intravenous of
}

Blood (ILIB) en Odontología: una revisión integradora

\author{
Maria Regina Almeida de Menezes \\ ORCID:https://orcid.org/0000-0003-3012-3979 \\ Universidade de Pernambuco, Brasil \\ E-mail: regina.menezes@upe.br \\ Esdras Gabriel Alves-Silva \\ ORCID:https://orcid.org/0000-0002-2309-1115 \\ Universidade Estadual de Campinas, Brasil \\ E-mail: esdras0702@yahoo.com.br \\ Emilene Correia Gomes de Santana \\ ORCID: https://orcid.org/0000-0002-7766-7116 \\ Universidade de Pernambuco, Brasil \\ E-mail: emilene.correia@upe.br \\ Vinícius Cristovão de Oliveira Mendes \\ ORCID: https://orcid.org/0000-0001-6983-1951 \\ Universidade de Pernambuco, Brasil \\ E-mail: viniciusmendes160502@outlook.com
}

\begin{abstract}
Resumo
O objetivo deste trabalho é responder à pergunta norteadora "quais os efeitos da irradiação do sangue por via intravenosa na odontologia?". Para isso, foi realizada uma busca mais direcionada e aprofundada nas plataformas Cochrane, SciELO, PubMed e Scopus através dos descritores "Low level laser; photobiomodulation; dentistry", que buscou integrar as palavras "and" e "more", com objetivo de selecionar artigos com tal conteúdo acima descrito. Por fim, após a realização dos critérios de elegibilidade, foram selecionados seis artigos, compostos no período de 2016 a Novembro de 2020, para a realização de uma análise mais aprofundada. Pois este assunto trata-se de algo novo, no entanto, sabese que, na Rússia, muitos estudos já foram publicados, porém sem tradução para língua inglesa. Assim, após a realização da leitura dos artigos selecionados, pode-se observar que a aplicação do laser de baixa intensidade com dosimetrias e sugestões adotadas é extremamente benéfica, mas o seu uso ainda necessita de estudos mais aprofundados devido aos efeitos colaterais que podem surgir, o que demonstra a presença de fatores positivos e negativos no uso dessa técnica, principalmente no uso odontológico. A limitação desse estudo está em identificar os descritores no DECs e encontrar artigos em língua inglesa e outras mais comuns, tornando-se necessário que estudos continuem sendo realizados e publicados, para que assuntos como esse sejam disseminados para a população.
\end{abstract}

Palavras-chave: Laser de baixa potência; Fotobiomodulação; Odontologia.

\begin{abstract}
The objective of this work is to answer the guiding question "what are the effects of blood irradiation intravenously in dentistry?". For this, a more targeted and in-depth search was performed on the Cochrane, SciELO, PubMed and Scopus platforms through the descriptors Low level laser; photobiomodulation; dentistry, which sought to integrate the words "and" and "more" in order to select articles with the content described above. Finally, after the fulfillment of the eligibility criteria, six articles were selected, composed in the period from 2016 to November 2020, to carry out a more in-depth analysis. As this subject is something new, however, it is known many studies have been published in Russia, but without an English translation. Thus, after reading the selected articles, it could be observed that the application of low-level laser with dosimetry and adopted suggestions is extremely beneficial, but its use still needs further studies due to the side effects that may arise, which demonstrates the presence of positive and negative factors in the use of this technique, mainly in dental use. The limitation of this study is in identifying the descriptors in the DECs and finding articles in English and other more common ones, making it necessary that studies continue to be carried out and
\end{abstract}


published, so that subjects like this are disseminated to the population.

Keywords: Low level laser; Photobiomodulation; Dentistry.

\section{Resumen}

El objetivo de este trabajo es responder a la pregunta orientadora "¿Cuáles son los efectos de la irradiación de sangre por vía intravenosa en odontología?". Para ello, se realizó una búsqueda más focalizada y profunda en las plataformas Cochrane, SciELO, PubMed y Scopus a través de los descriptores "Láser de bajo nivel; fotobiomodulación; odontología ", que buscaba integrar las palabras "Y" y "MÁS" para seleccionar artículos con el contenido descrito anteriormente. Finalmente, luego del cumplimiento de los criterios de elegibilidad, se seleccionaron seis artículos, compuestos en el período de 2016 a noviembre de 2020, para realizar un análisis más profundo, ya que este tema es algo nuevo, sin embargo, sabemos Se han publicado muchos estudios en Rusia, pero sin traducción al inglés. Así, luego de leer los artículos seleccionados, se puede observar que la aplicación de láser de bajo nivel con dosimetría y sugerencias adoptadas es sumamente beneficiosa, pero su uso aún requiere más estudios debido a los efectos secundarios que pueden surgir. lo que demuestra la presencia de factores positivos y negativos en el uso de esta técnica, principalmente en uso odontológico. La limitación de este estudio está en identificar los descriptores en las DEC y encontrar artículos en inglés y otros más comunes, por lo que es necesario que se sigan realizando y publicando estudios, para que temas como este se difundan a la población.

Palabras clave: Láser de baja intensidad; Fotobiomodulación; Odontología.

\section{Introdução}

A terapia por meio do ILIB (do inglês “Intravascular Laser Irradiation of Blood”), também chamada de laserterapia e terapia de fotobiomodulação (do inglês "Photobiomodulation Therapy" ou "PBMT"), utiliza a luz não ionizante, ou infravermelha, para estimular a região a nível sistêmico (Cronshaw, et al., 2020) - por meio da aplicação na artéria radial; seu uso estimula a microcirculação, que é resultado de um aumento na produção de trifosfato de adenosina (ATP), óxido nítrico (NO) e reativas espécies de oxigênio (ROS). Ela pode ser utilizada no campo odontológico isoladamente ou em parceria com outros tratamentos.

Dessa forma, ocorre um auxílio no tratamento de úlceras aftosas, hipersensibilidade dentinária e mitigação da mucosite oral (Cronshaw, et al., 2020), essa ocasionada pela radioterapia no tratamento de câncer de cabeça e pescoço (Zecha, et al. 2016), bem como a eliminação de microorganismos provocados pelo fungo Candida albicans (Cerdeira, et al., 2016) e aplicações pósoperatórias na osseointegração e estabilidade de implantes dentários, que, possivelmente, potencializam efeitos positivos (Zayed, et al., 2020). Assim, evidenciam-se os benefícios no auxílio ao conforto paciente e, também, do dentista, visto que essa é uma terapia indolor em comparação com a aplicação de lasers de alta intensidade, esses responsáveis pela emissão de efeitos térmicos muitas vezes indesejáveis.

Nesse contexto, percebe-se que a terapia de baixa intensidade necessita de um comprimento de onda 633 e $685 \mathrm{~nm}$ ou 780-830 nm; densidade de energia, saída de laser ou diodo emissor de luz (LED) entre 10 e 150 mW; dose, 2-3 J (J / cm 2), e não mais do que $6 \mathrm{~J} / \mathrm{cm} 2$ na superfície do tecido tratado (Zecha, et al., 2016). Contudo, caso as medidas de dosimetrias sugeridas não sejam adotadas, diversas complicações, possivelmente associadas ao uso da irradiação no tratamento das mucosites orais advindas como efeitos colaterais do tratamento do câncer da cabeça e pescoço (Ibidem, 2016), além de úlceras aftosas e outras enfermidades, podem surgir, tais como dermatite, disfagia, boca seca, disgeusia, trismo, necrose e alterações da fala. Desse modo, torna-se explícita a necessidade de um manejo e dosimetria adequados para a realização do tratamento das disfunções apresentadas, para que não haja uma debilitação do paciente em outros fatores.

Diante disso, compreende-se que, devido aos comprimentos de onda serem baixos, a técnica referida não é considerada invasiva, o que destina conforto ao paciente devido às propriedades anti-inflamatórias, analgésicas e cicatrizantes associadas, que atuam em conjunto para a destinação de um tratamento benéfico.

Nesse sentido, as limitações encontradas neste presente estudo resumiram-se em encontrar os descritores oficiais no site

DECs e promover uma associação eficaz, utilizando os ligantes “AND" e "MORE”, para que os artigos fossem encontrados e 
analisados para compor com excelência o conteúdo dessa revisão integrativa. Isso comprova a necessidade de estudos mais aprofundados e completos sobre esse tema, que permitiriam, assim, uma disseminação maior de informações para a população.

Por fim, é interessante pontuar que a relevância desse estudo está na presença de plataformas de alto impacto, que foram utilizadas para a obtenção dos artigos por meio de uma busca com os descritores oficiais. Ademais, este trabalho foi realizado com o auxílio de mais de um artigo os quais contêm opiniões muitas vezes convergentes, mas também divergentes, o que resultou em um estudo clínico randomizado dos benefícios e limitações da irradiação com laser de baixa intensidade (ILIB) na Odontologia.

\section{Metodologia}

O presente estudo trata-se de uma revisão integrativa da literatura realizada por meio de uma busca de artigos científicos na base de dados eletrônicos PubMed, Cochrane Library, SciELO e Scopus, utilizando os descritores "Low level laser AND photobiomodulation MORE dentistry". Nesse viés, combinando as associações e desfechos de interesse, foi elaborada a descrita Figura 1 presente na página a seguir.

Figura 1. Fluxograma de estratégia de busca nas bases de dados.

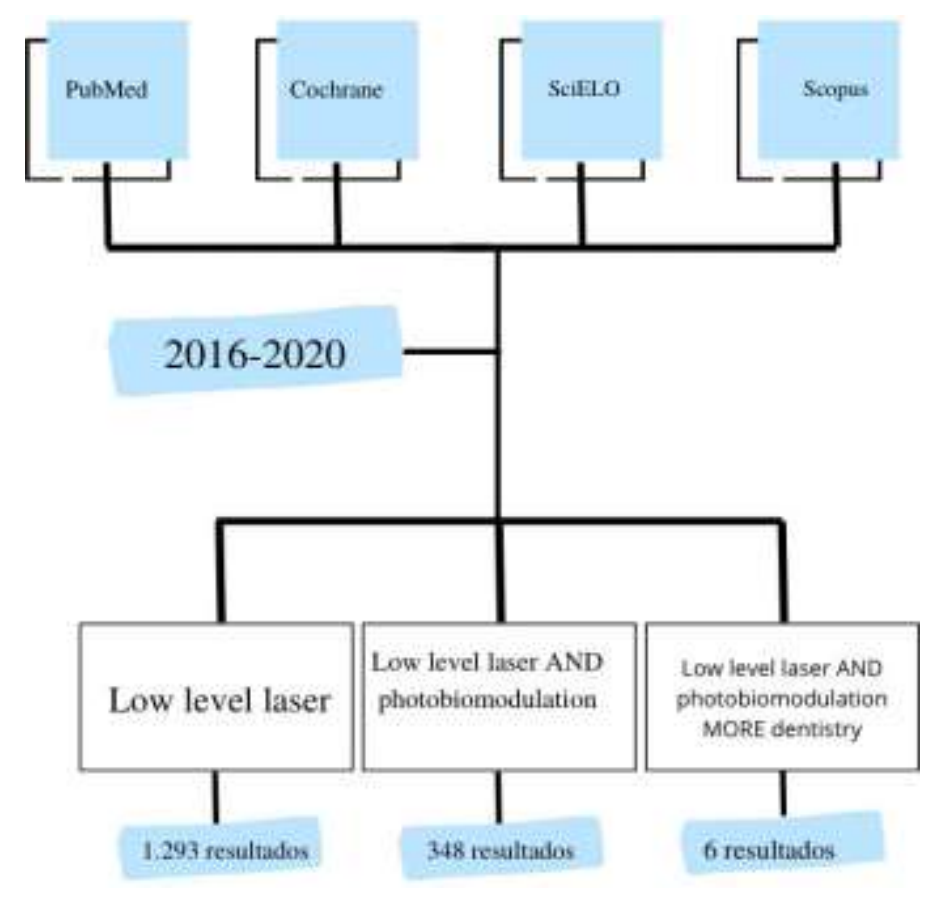

Fonte: Autores (2020).

A partir da busca de artigos científicos nas plataformas acima mencionados no período de 2016 a 2020, utilizando o descritor "Low level laser" obteve-se um total de 1.293 resultados, após a junção deste descritor com "photobiomodulation" foram filtrados 348 resultados, que já apareciam na pesquisa com apenas o primeiro descritor. Após a junção dos descritores anteriores com "dentistry", obteve-se uma amostra de 6 artigos, também já apresentados nas duas outras pesquisas. Assim, concluiu-se que os seis artigos resultantes estavam presentes na plataforma PubMed. 
O processo de análise para avaliação e seleção dos artigos foi realizado por dois pesquisadores, de forma conjunta, para que as informações presentes nesta revisão integrativa estivessem de acordo. Em casos de divergências ou dúvidas quanto à inclusão dos artigos, houve a participação de um terceiro pesquisador avaliador. Nesse sentido, a seleção das publicações foi conduzida em duas fases: (1) leitura dos resumos, e (2) análise qualitativa dos textos na íntegra.

Para seleção das fontes, foram consideradas como critérios de inclusão os artigos originais disponibilizados na íntegra, escritos no idioma inglês, indexados no período de 2016 a 2020, que tratavam de estudos in vitro, ex vivo e relatos de caso. Não foram incluídos artigos publicados em outras línguas ou em um período diferente do delimitado. Nesse contexto, pode-se notar a seguinte Figura 2, que ilustra a estratégia de busca de acordo com os critérios citados.

Figura 2. Fluxograma de estratégia de busca e seleção dos artigos.

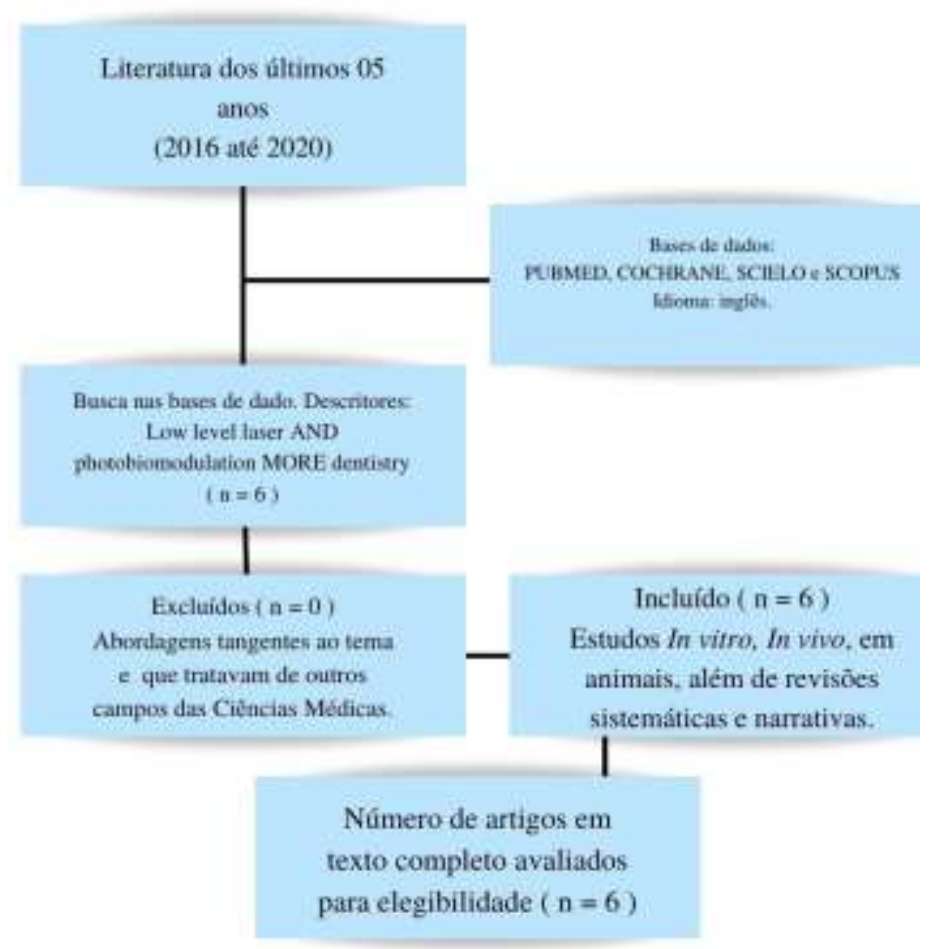

Fonte: Autores (2020).

De acordo com esse quadro, foi realizada uma leitura completa dos estudos encontrados, chegando a uma amostra final de seis artigos incluídos nesta revisão. Além disso, não foram utilizadas teses, dissertações e monografias, visto que a realização de uma busca desses estudos é inviável logisticamente e as bases de dados utilizadas não têm esse tipo de publicação.

\section{Resultados}

Esse estudo visou analisar a literatura acerca do uso de lasers de baixa potência, observando os benefícios, as limitações, as relevâncias e os impactos do uso na área odontológica. A presente revisão integrativa foi capaz de encontrar seis estudos recentes que demonstram a atuação do laser infravermelho, ou seja, de baixa potência, na odontologia. 
Research, Society and Development, v.10, n.2, e30910212576, 2021

(CC BY 4.0) |ISSN 2525-3409 | DOI: https://dx.doi.org/10.33448/rsd-v10i2.12576

Quadro 1. Seleção dos artigos de acordo com o autor, tipo de estudo, objetivos, metodologias, resultados e conclusão.

\begin{tabular}{|c|c|c|c|c|}
\hline Autores e estudo & Objetivo & Metodologia & Resultado & Conclusão \\
\hline $\begin{array}{l}\text { Mark Cronshaw, et al., } \\
\text { (2020) } \\
\text { Estudos in vitro e em } \\
\text { animais. }\end{array}$ & $\begin{array}{l}\text { Essa é uma revisão sistemática e meta- } \\
\text {-análise de publicações randomizadas } \\
\text { controladas para examinar uma } \\
\text { possível relação entre o tamanho do } \\
\text { ponto óptico no tecido de superfície e a } \\
\text { irradiação recebida. }\end{array}$ & $\begin{array}{l}\text { Utilizou-se o PubMed, Cochrane, } \\
\text { Database of Reviews e os } \\
\text { motores de busca do Google } \\
\text { Scholar para identificar ensaios } \\
\text { clínicos humanos de terapia PBM } \\
\text { em odontologia em um total de } \\
766 \text { artigos entre fevereiro de } \\
2009 \text { e junho de } 2020 \text {. }\end{array}$ & $\begin{array}{l}\text { Após uma avaliação detalhada dos } \\
\text { fatores constatou que tecidos alvos } \\
\text { maiores são considerados mais } \\
\text { responsivos a terapia por uso de um } \\
\text { maior ponto de superfície óptica }\end{array}$ & $\begin{array}{l}\text { Muitos fatores podem variar o sucesso clínico } \\
\text { pela individualidade de cada sujeito. }\end{array}$ \\
\hline $\begin{array}{l}\text { Cláudio Daniel Cerdeira, et al., } \\
\qquad \begin{array}{l}(2016) \\
\text { Estudo in vivo. }\end{array}\end{array}$ & $\begin{array}{l}\text { A terapia a laser de baixo nível } \\
\text { (LLLT) é conhecida por aumentar a } \\
\text { transferência de elétrons mitocondrial } \\
\text { e perguntando-se se poderia estimular } \\
\text { a oxidação de neutrófilos e aumentar a } \\
\text { capacidade de matar microorga- } \\
\text { nismos. }\end{array}$ & $\begin{array}{l}\text { Sangue de humanos saudáveis } \\
\text { foram coletados e PMN foram } \\
\text { isolados das amostras. PMN } \\
\text { foram tratados in vitro com luz } \\
\text { laser CW de } 660 \text { ou } 780 \mathrm{~nm} \text { e } \\
\text { subsequen- temente incubados } \\
\text { com células de Candida } \\
\text { albicans. }\end{array}$ & $\begin{array}{l}\text { Geração de radicais hidroxila, hipoclo- } \\
\text { rito, ânions e ânions superóxido foram } \\
\text { verificados ao utilizar as luzes, em que } \\
\text { a luz } 660 \mathrm{~nm} \text { foi mais eficaz que a de } \\
780 \mathrm{~nm} \text { no aumento estouro } \\
\text { respiratório por PMN. }\end{array}$ & $\begin{array}{l}\text { A terapia a laser de baixo nível (LLLT) excitou o } \\
\text { PMN para um perfil funcional superior, que foi } \\
\text { traduzido como uma produção superior de } \\
\text { Espécies reativas de oxigênio (ROS), como } \\
\text { radicais hidroxila }(\mathrm{HO} \cdot \text { ) e ânions hipoclorito } \\
(\mathrm{ClO}-\text { ) e aumento capacidade fungicida contra } \\
\text { células de Candida albicans. }\end{array}$ \\
\hline $\begin{array}{l}\text { Zecha, et. al., } \\
\qquad \text { (2016) } \\
\text { Estudos clínicos. }\end{array}$ & $\begin{array}{l}\text { Muitas evidências apontam para a } \\
\text { eficácia da terapia a laser de baixa } \\
\text { potência (LLLT) para tratamento de } \\
\text { mucosite oral em pacientes submetidos } \\
\text { a radioterapia para câncer de cabeça e } \\
\text { pescoço (HNC). }\end{array}$ & $\begin{array}{l}\text { Este estudo é uma revisão } \\
\text { narrativa não sistemática. }\end{array}$ & $\begin{array}{l}\text { Revisamos os mecanismos de ação do } \\
\text { PBM e as considerações dosimétricas. } \\
\text { Embora não haja descrições anedóticas } \\
\text { de maus resultados tumorais em } \\
\text { pacientes tratados com } \text { PBM } \\
\text { confirmando sua a neutralidade com } \\
\text { relação à resposta ao câncer é uma } \\
\text { prioridade crítica. }\end{array}$ & $\begin{array}{l}\text { Baseado em seus efeitos terapêuticos, o PBM tem } \\
\text { uma grande importância em vários } \\
\text { medicamentos. Embora as evidências sugiram } \\
\text { que PBM usando LLLT é seguro em pacientes } \\
\text { com HNC ,mais pesquisas são necessárias para } \\
\text { detectar quaisquer efeitos adversos do PBM no } \\
\text { tratamento do câncer e sobrevivência. }\end{array}$ \\
\hline
\end{tabular}


Research, Society and Development, v.10, n.2, e30910212576, 2021

(CC BY 4.0) |ISSN 2525-3409 | DOI: https://dx.doi.org/10.33448/rsd-v10i2.12576

\begin{tabular}{|c|c|c|c|c|}
\hline $\begin{array}{l}\text { Zecha, et al., } \\
\qquad \text { (2016) } \\
\text { Estudos clínicos. }\end{array}$ & $\begin{array}{l}\text { Várias são as evidências que apoiam a } \\
\text { eficácia da terapia a laser de baixa } \\
\text { potência para o manejo da mucosite } \\
\text { oral em pacientes submetidos à } \\
\text { radioterapia para câncer de cabeça e } \\
\text { pescoço(HNC). Avanços recentes da } \\
\text { tecnologia PBM, possibilitam um } \\
\text { melhor gerenciamento de uma gama } \\
\text { mais ampla de complicações associadas } \\
\text { ao HNC. Aumentando a adesão do } \\
\text { paciente ao tratamento. }\end{array}$ & $\begin{array}{l}\text { A revisão narrativa e a } \\
\text { apresentação dos parâmetros de } \\
\text { PBM são baseadas em evidências } \\
\text { e na opinião de especialistas. }\end{array}$ & $\begin{array}{l}\text { O PBM pode ter aplicações potenciais na } \\
\text { gestão de uma ampla gama de efeitos da } \\
\text { radioterapia (CRT) em pacientes em } \\
\text { tratamento para HNC. }\end{array}$ & $\begin{array}{l}\text { O PBM pode ter um papel no tratamento de } \\
\text { suporte para uma ampla gama de complicações } \\
\text { associadas ao tratamento de HNC com CRT. A } \\
\text { irradiação PBM sugerida e dosimétrica } \\
\text { parâmetros, que são potencialmente eficazes para } \\
\text { essas complicações, destinam-se a fornecer } \\
\text { orientações para estudos futuros bem elaborados. }\end{array}$ \\
\hline $\begin{array}{l}\text { Zayed, et al., } \\
\qquad(2020) \\
\text { Estudo in vitro e em animais. }\end{array}$ & $\begin{array}{l}\text { O objetivo foi avaliar a eficácia clínica } \\
\text { do PBM na osseointegração de } \\
\text { implantes dentários. }\end{array}$ & $\begin{array}{l}\text { Dois revisores realizaram de } \\
\text { forma independente uma busca } \\
\text { eletrônica abrangente de artigos } \\
\text { publicados até } 10 \text { de janeiro de } \\
2020 \text {, nos bancos de dados } \\
\text { PubMed e Cochrane Library. } \\
\text { Devido à heterogeneidade } \\
\text { substancial,apenas uma síntese } \\
\text { narrativa dos estudos incluídos é } \\
\text { apresentada. }\end{array}$ & $\begin{array}{l}\text { Sete estudos clínicos relevantes foram } \\
\text { incluídos e eles usaram vários } \\
\text { parâmetros e dispositivos PBM. } \\
\text { Quatro estudos descobriram que o } \\
\text { PBM tem um potencial efeito positivo } \\
\text { sobre o resultado da estabilidade do } \\
\text { implante dentário enquanto três } \\
\text { estudos relataram que o PBM não tem } \\
\text { efeito sobre o implante de estabilidade }\end{array}$ & $\begin{array}{l}\text { Os resultados desta revisão sistemática sugerem } \\
\text { que a aplicação pós-operatória de PBM pode } \\
\text { potencialmente obter algum efeito positivo na } \\
\text { osseointegração e estabilidade do implante } \\
\text { dentário. No entanto, adicionais estudos são } \\
\text { necessários com uniformidade nos métodos para } \\
\text { fornecer uma avaliação mais robusta desse efeito. }\end{array}$ \\
\hline $\begin{array}{l}\text { Wang, et al., } \\
\qquad \text { (2016) } \\
\text { Estudo in vitro. }\end{array}$ & $\begin{array}{l}\text { As células- tronco derivadas do tecido } \\
\text { adiposo humano tem o potencial de se } \\
\text { diferenciar em vários tipos celulares } \\
\text { incluindo osteoblastos. }\end{array}$ & $\begin{array}{l}\text { Fotobiomodulação }(\mathrm{PBM}) \\
\text { Usando comprimentos de onda } \\
\text { infravermelho foram relatados } \\
\text { efeitos de proliferação } \\
\text { osteogênica e diferenciação } \\
\text { células tronco. }\end{array}$ & $\begin{array}{l}\text { Os comprimentos de onda de } 420 \text { e } \\
550 \mathrm{~nm} \text { foram mais eficazes na } \\
\text { estimulação da diferenciação de } \\
\text { osteoblastos em comparação com } 660 \\
\text { nm e } 810 \mathrm{~nm} \text {. O cálcio intracelular foi } \\
\text { maior após } 420 \mathrm{~nm} \text { e } 540 \mathrm{~nm} \text {, e pode } \\
\text { ser inibido pela capsazepina e } \\
\text { SKF96365, que também inibiu a } \\
\text { diferenciação osteogênica. }\end{array}$ & $\begin{array}{l}\text { Nossa hipótese é que a ativação da luz canais íon } \\
\text { de cálcio bloqueados por luz azul e verde podem } \\
\text { explicar nossos resultados. }\end{array}$ \\
\hline
\end{tabular}

Fonte: Autores (2020) 
Foram selecionados, dentre os critérios, seis artigos. Estes abordaram o uso da irradiação com laser de baixa intensidade (ILIB) mediante estudos in vitro, in vivo, e em animais, para a obtenção de resultados que auxiliaram na área odontológica. Assim, nota-se que são necessárias um número maior de pesquisas clínicas, em especial randomizadas, com um maior número de pacientes e aplicações para obterem-se conclusões mais significativas sobre as aplicações do laser não apenas no contexto local - ao referido ILIB, mas também no regional e sistêmico.

\section{Discussão}

Com isso, pôde-se observar a existência não apenas de diversos fatores que contribuíram de maneira positiva para o uso benéfico da irradiação com laser de baixa potência no campo odontológico, mas também a presença de limitações que prejudicam a utilização dessa ferramenta. Ademais, não foram excluídos artigos ao final da busca, pois já estavam coerentes com os critérios de inclusão. Assim, torna-se importante ressaltar a opinião de autoridade dos autores para a efetivação de conclusões eficazes e coerentes para a temática abordada.

Em primeiro plano, cabe abordar os benefícios relacionados ao laser de baixa potência mediante o uso na cabeça e pescoço. Nesse contexto, de acordo com Cronshaw, et al. (Ibidem, 2020), em seu estudo em organismo in vitro e em animais, o uso da irradiação de baixa intensidade está associada à mitigação de feridas na região da boca, redução da inflamação - devido à inibição da ciclooxigenase e, consequentemente, da resposta inflamatória - além de apresentar uma correlação cujo especificou que o sucesso de aplicação do laser de baixa potência está relacionada com o tamanho da superfície, ou seja, deve ser pequena para o resultado ser satisfatório. Por outro lado, Cerdeira, et al. (Ibid, 2016), buscou apresentar a terapia a laser de baixo nível como uma solução para a esterilização do orifício bucal contra a bactéria Candida albicans, mostrando-se eficaz para a utilização em proximidades futuras. $\mathrm{O}$ estudo foi realizado em organismos in vitro e a luz aplicada apresentou uma potência de $40 \mathrm{~mW}$ (Megawatt) e energia total de 19,2 J (Joules).

Além disso, é perceptível que a fotobiomodulação, de acordo com Zecha, et al. (Ibidem, 2016), em seus dois artigos partes 1 e 2 - que foram objetos de estudo para a composição do presente trabalho, apresenta benefícios relacionados à mitigação da mucosite oral em pacientes com câncer de cabeça e pescoço que foram submetidos à radioterapia. Já o autor Zayed, et al. (Ibid, 2020), apresenta em seu estudo a aplicação pós-operatória da laserterapia como positiva na osseointegração e estabilidade de implantes dentários. Por fim, Wang, et al. (Ibid, 2016), apresentou o uso do laser (azul e verde) como contribuinte para a diferenciação de células-tronco osteogênicas do tecido adiposo, o que pode ser benéfico na utilização de procedimentos ortopédicos por meio do uso de enxerto ósseo. Posto isso, percebe-se que os efeitos relacionados às capacidades antiinflamatórias, analgésicas e cicatrizantes encontram semelhanças mediante a aplicação do laser e resultados somáticos, mas também é interessante analisar as limitações presentes nesta ferramenta à base de luz que é, na maioria dos casos, composta por laser de diodo.

Nessa perspectiva, em segundo plano, é evidente que a irradiação com laser de baixa intensidade apresenta também limitações relacionadas ao seu uso, seja na requisição de uma dosimetria específica (Zecha, 2016), seja na carência de estudos sobre esse o campo em questão (Zayed, 2020). Outrossim, Zecha et. al (Ibidem, 2016) relacionou a aplicação do laser dosimetria, protocolos de tratamento, mecanismos indesejados de ação - com o surgimento de dúvidas relacionadas à eficácia do tratamento no contexto de aplicação da técnica sem a presença de alguns desses mecanismos. Outrossim, o laser vermelho obteve resultados inferiores em comparação com as luzes azul e verde mediante a promoção de diferenciação osteogênica, o que comprova a limitação desse em relação a este. Dessa forma, comprova-se algumas das limitações de acordo com os próprios autores, temática que carece de estudos mais aprofundados sobre os empecilhos encontrados. 
A relevância deste estudo está na realização de busca aprofundada nas plataformas de literatura de alto impacto por meio de descritores oficiais do banco de dados DECs; além da presença de alguns autores com alto grau de reconhecimento, o que impõem ao artigo apresentado um alto grau de organização e relevância no âmbito científico. Além disso, essa é uma temática que necessita de artigos e revisões literárias atualizadas, de modo que a literatura atual carece de estudos nesse aspecto sobre o uso da irradiação com lasers de baixa intensidade (ILIB) na Odontologia.

Os impactos deste artigo não se restringem apenas à promoção de uma atualização da literatura atual, mas também contribui para a disseminação do conhecimento para a comunidade acadêmica e civil sobre o uso dos lasers na Odontologia, seja a nível sistêmico, seja a nível local. Assim, torna-se indispensável que mais estudos randomizados sejam realizados e publicados para que a informação seja disseminada; além de popularizar o uso dos lasers de baixa potência no campo odontológico, visto que é um método minimamente invasivo e indolor.

\section{Conclusão}

Por fim, tornam-se evidentes os benefícios, limitações, impactos e relevâncias da aplicação do Irradiation Laser Intravenous of Blood (ILIB) na Odontologia, visto que sua potência entra como critério de elegibilidade igual aos demais lasers de baixa potência. Além do efeito causado no organismo ser não apenas sistêmico, mas também local, o que auxilia no tratamento de diversas disfunções orais discutidas acima.

Desse modo, é visível que mais pesquisas precisam ser desenvolvidas para suprir a ausência de mais conteúdos da literatura sobre a temática abordada, pois a maior parte dos artigos relacionados com a temática ILIB encontram-se em russo e datados de anos anteriores ao de 2016. O que comprova, dessa maneira, a importância de atualizar o tema por meio, também, de estudos clínicos randomizados.

Posto isso, percebe-se que, como sugestão, vê-se a necessidade de um maior investimento na área clínica odontológica por meio da aquisição do ILIB nas universidades e campos de estudos, para que, assim, os efeitos dessa técnica possam ser acompanhados com mais eficácia e, consequentemente, mais conteúdos sobre essa temática sejam produzidos pelos futuros autores e seus orientadores.

\section{Referências}

Cerdeira, C. D., \& Lima Brigagão, M. R., \& Carli, M. L., et al. (2016). Low-level laser therapy stimulates the oxidative burst in human neutrophils and increases their fungicidal capacity. J Biophotonics, 9(11-12), 1180-1188. 10.1002/jbio.201600035

Cronshaw, M., \& Parker, S., \& Anagnostaki, E., \& Mylona, V., \& Lynch, E., \& Grootveld, M. (2020). Photobiomodulation Dose Parameters in Dentistry: A Systematic Review and Meta-Analysis. Dentistry Journal, 8(4), 114.

Descritores em Ciências da Saúde: DeCS. (2020). BIREME / OPAS / OMS, 2017.

Pereira, A. S. et al. (2018). Metodologia da pesquisa científica. UFSM.

Wang, Y., \& Huang, Y. Y., \& Wang, Y., \& Lyu, P., \& Hamblin, M. R. (2016). Photobiomodulation (blue and green light) encourages osteoblastic-differentiation of human adipose-derived stem cells: role of intracellular calcium and light-gated ion channels. Sci Rep. 6, 33719. 10.1038/srep33719.

Zayed, S. M., \& Hakim, A. A. A. (2020). Clinical Efficacy of Photobiomodulation on Dental Implant Osseointegration: A Systematic Review. Saudi J Med Med Sci. 8(2), 80-86. 10.4103/sjmms.sjmms_410_19.

Zecha, J. A., \& Raber-Durlacher, J. E., \& Nair, R. G., \& Epstein, J. B., \& Sonis, S. T., \& Elad, S., \& Hamblin, M. R., \& Barasch, A., \& Migliorati, C. A., \& Milstein, D. M., \& Genot, M. T., \& Lansaat, L., \& van der Brink, R., \& Arnabat-Dominguez, J., \& van der Molen, L., Jacobi, I., \& van Diessen, J., \& de Lange, J., \& Smeele, L. E., \& Schubert, M. M., \& Bensadoun, R. J. (2016). Low level laser therapy/photobiomodulation in the management of side effects of chemoradiation therapy in head and neck cancer: part 1: mechanisms of action, dosimetric, and safety considerations. Supportive care in cancer: official journal of the Multinational Association of Supportive Care in Cancer. 24(6), 2781-2792. https://doi.org/10.1007/s00520-016-3152-z.

Zecha, J. A., \& Raber-Durlacher, J. E., \& Nair, R. G., \& Epstein, J. B., \& Elad, S., \& Hamblin, M. R., \& Barasch, A., \& Migliorati, C. A., \& Milstein, D. M., \& Genot, M. T., \& Lansaat, L., \& van der Brink, R., \& Arnabat-Dominguez, J., \& van der Molen, L., \& Jacobi, I., \& van Diessen, J., \& de Lange, J., \& Smeele, L. 
Research, Society and Development, v.10, n.2, e30910212576, 2021

(CC BY 4.0) |ISSN 2525-3409 | DOI: https://dx.doi.org/10.33448/rsd-v10i2.12576

E., \& Schubert, M. M., \& Bensadoun, R. J. (2016) Low-level laser therapy/photobiomodulation in the management of side effects of chemoradiation therapy in head and neck cancer: part 2: proposed applications and treatment protocols. Supportive care in cancer: official journal of the Multinational Association of Supportive Care in Cancer. 24(6), 2793-2805. https://doi.org/10.1007/s00520-016-3153-y. 